\title{
Correction to: Quasicompact and Riesz Composition Endomorphisms of Lipschitz Algebras of Complex-Valued Bounded Functions and Their Spectra
}

\author{
Maliheh Mayghani1 $^{1}$ - Davood Alimohammadi ${ }^{2}$
}

Published online: 16 May 2018

(C) Iranian Mathematical Society 2018

\section{Correction to: Bull. Iran. Math. Soc. https://doi.org/10.1007/s41980-018-0021-1}

In the original publication of this article, the e-mail address of the corresponding author was inadvertently entered incorrectly.

The correct e-mail address is: d-alimohammadi@araku.ac.ir

Please excuse this mistake.

The original article can be found online at https://doi.org/10.1007/s41980-018-0021-1.

$凶$ Davood Alimohammadi

d-alimohammadi@araku.ac.ir

Maliheh Mayghani

m_maighany@yahoo.com

1 Department of Mathematics, Payame Noor University (PNU), P.O. Box 19395-3697, Tehran, Iran

2 Department of Mathematics, Faculty of Science, Arak University, Arak 38156-8-8349, Iran 